\title{
Prognostic value of CEA and CA19-9 in patients with local advanced rectal cancer receiving neoadjuvant chemoradiotherapy, radical surgery and postoperative chemotherapy
}

\author{
Jingjing Shan ${ }^{\#}$, Benxing Gu${ }^{\#}$, Liming Shi, Xuanxuan Wang, Wenyuan Ye, Weiwen Zhou, Xiaonan Sun^ \\ Department of Radiation Oncology, Sir Run Run Shaw Hospital, School of Medicine, Zhejiang University, Hangzhou, China \\ Contributions: (I) Conception and design: J Shan, B Gu, X Sun; (II) Administrative support: X Sun; (III) Provision of study materials or patients: \\ J Shan, L Shi, X Wang; (IV) Collection and assembly of data: W Ye, W Zhou; (V) Data analysis and interpretation: J Shan, B Gu, X Sun; (VI) \\ Manuscript writing: All authors; (VII) Final approval of manuscript: All authors. \\ \#These authors contributed equally to this work. \\ Correspondence to: Xiaonan Sun. Department of Radiation Oncology, Sir Run Run Shaw Hospital, School of Medicine, Zhejiang University, 3 East \\ Qingchun Road, Hangzhou 310016, China. Email: sunxiaonan@zju.edu.cn.
}

Background: We aim to investigate the prognostic factors and evaluate the role of carcinoembryonic
antigen (CEA) and carbohydrate antigen 19-9 (CA19-9) in local advanced rectal cancer (LARC) patients who
received neoadjuvant chemoradiotherapy (neo-CRT), radical surgery and postoperative chemotherapy.
Methods: In total, 197 cases of LARC patients who underwent neo-CRT, total mesorectal excision (TME),
and adjuvant chemotherapy were recruited. Serum levels of CEA and CA19-9 were detected both at baseline
and after neo-chemoradiotherapy. Multivariate analysis was used to assess correlations between levels of CEA
and CA19-9 and patients' prognosis (survival, recurrence, and metastasis). Rates of survival, distant metastasis
(DM), and local recurrence (LR) were estimated using Kaplan-Meier survival analysis, the log-rank test, and
Cox proportional hazards.

Results: The median follow-up time was 45.3 months, and a cohort of 197 patients was analyzed; 84 (42.6\%) patients had elevated baseline CEA levels, 21 (10.7\%) patients had elevated baseline CA19-9 levels, and 14 (7.1\%) patients had both; 77.4\% (65/84) patients with high CEA levels and 76.2\% (16/21) with high CA199 levels returned to normal after neo-chemoradiotherapy. The Cox regression model suggested that elevated CEA was associated with an increased risk of disease-free survival (DFS) (HR: 2.058, 95\% CI: 1.034-4.096, $\mathrm{P}=0.040$ ) and DM (HR: 2.144, 95\% CI: 1.058-4.346, $\mathrm{P}=0.034)$. Elevated CA19-9 was identified as an independent prognostic factor, with poorer overall survival (OS) (HR: 2.894, 95\% CI: 1.196-7.006, P=0.018) and DFS (HR: 4.533, 95\% CI: 2.067-9.940, P<0.001) and increased incidences of LR (HR: 6.139, 95\% CI: 1.813-20.783, P=0.004) and DM (HR: 4.052, 95\% CI: 1.892-8.678, P<0.001). Besides, combined CEA with CA19-9 was a stronger prognostic predictor. Patients with both high levels of CEA and CA19-9 had the poorest DFS (HR: 8.157, 95\% CI: 3.232-20.591, P<0.001) and the highest risk of DM (HR: 8.790, 95\% CI: 3.324-23.248, $\mathrm{P}<0.001)$.

Conclusions: LARC patients with high levels of CEA or/and CA19-9 at initial treatment have a worse prognosis, even after neo-CRT, subsequent radical resection, and adjuvant chemotherapy. These findings suggest that this subset of patients requires more intensive treatment or additional treatment strategies.

Keywords: Carbohydrate antigen 19-9 (CA19-9); carcinoembryonic antigen (CEA); neoadjuvant chemoradiotherapy (neo-CRT); prognosis; rectal cancer

^ ORCID: Jingjing Shan: 0000-0001-8138-0858; Xiaonan Sun: 0000-0002-4027-4191. 
Submitted Jun 05, 2020. Accepted for publication Nov 16, 2020.

doi: 10.21037/tcr-20-2269

View this article at: http://dx.doi.org/10.21037/tcr-20-2269

\section{Introduction}

Neoadjuvant chemoradiotherapy (neo-CRT) combined with radical surgery and postoperative chemotherapy is recommended as the standard treatment of patients with stage II/III rectal cancer (1-3). With the multimodality treatment, the 5-year overall survival (OS) has exceeded $75 \%$ (4-6). Although, multidisciplinary treatment of local advanced rectal cancer (LARC) successfully improves outcomes, $5-10 \%$ of patients experiencing local recurrence (LR), and up to $30 \%$ suffering from distant metastasis (DM) $(7,8)$. Carcinoembryonic antigen (CEA) and Carbohydrate antigen 19-9 (CA19-9) are the most commonly used markers for diagnosis, treatment response assessment, and recurrence monitoring in CRC (9-13). Several studies have shown the prognostic value of CEA/CA19-9 in rectal cancer (14-17). However, few studies explored the correlations between elevated CEA and CA19-9 at initial treatment and prognosis in LARC patients after neo-CRT, total mesorectal excision (TME), and postoperative adjuvant chemotherapy.

Herein, we hypothesized that elevated CEA or/and CA19-9 might indicate poor prognosis in LARC patients after the standard treatment. Thus, we collected serum levels of CEA and CA19-9 at initial therapy and after neoCRT. Then, we investigated the correlations between elevated CEA and CA19-9 levels before treatment and prognosis (survival, recurrence, and metastasis) in patients with LARC after neo-CRT, TME, and postoperative adjuvant chemotherapy. We committed to finding highrisk prognostic factors in LARC patients after the standard first-line treatment. Moreover, we explored whether the management for the patient population needs improvement.

We present the following article in accordance with the REMARK reporting checklist (available at http://dx.doi. org/10.21037/tcr-20-2269).

\section{Methods}

\section{Patients}

Between June 2011 and September 2015, eligible patients aged at least 18 years, with clinical stage II $\left(\mathrm{T}_{3}{ }_{4} \mathrm{~N}_{0}\right)$ or III $\left(\mathrm{T}_{1-4} \mathrm{~N}_{1-2}\right)$ rectal adenocarcinoma who had a distal tumor border within $12 \mathrm{~cm}$ of the anal verge by colonoscopy were retrospectively collected at Sir Run Run Shaw Hospital, Zhejiang University School of Medicine, Hangzhou, China. This study was conducted according to the Declaration of Helsinki (as revised in 2013), and the ethic committee of Sir Run Run Shaw Hospital, School of Medicine, Zhejiang University (Project number 20180927-1) approved the study. All patients provided written informed consent before the treatment.

Enhanced magnetic resonance imaging (MRI) and endorectal ultrasound were used for local staging. A full colonoscopy or rigid proctoscopy and computed tomography (CT) scan of the chest and abdomen were performed before treatment. Positron emission tomography-CT (PET-CT) was recommended to exclude $\mathrm{DM}$ if there were suspicious test results. Patients were required to have an Eastern Cooperative Oncology Group performance status $\leq 1$ and adequate hematologic (defined as hemoglobin $\geq 90 \mathrm{~g} / \mathrm{L}$, neutrophils $\geq 1.5$ billion/L, platelets $\geq 80,000$ billion/L), liver function (total bilirubin $<2 \mathrm{mg} / \mathrm{dL}$; alanine aminotransferase, aspartate aminotransferase $<3 \times$ the upper limit of normal), and renal function (creatinine $\leq 130 \mu \mathrm{mol} / \mathrm{L}$ ). Exclusion criteria included the presence of metastatic disease, previously receiving radiotherapy or chemotherapy to the pelvis, the presence of previous or concurrent cancers, or clinically significant cardiac disease.

\section{Treatment}

Preoperative radiotherapy consisted of 45-50 Gy administered in 25 fractions delivered with a minimum energy of 6-MV photons. The target delineation criteria refer to the North American Tumor Radiation Therapy Collaborative Group (RTOG) and the European Society of Oncology (ESMO) guidelines. Organs at risk included the small intestine, colon, bladder, and femur head, and corresponding dose limitations were as follows: small intestine $\mathrm{D}_{\max }<52 \mathrm{~Gy}$; colon $\mathrm{D}_{\max }<54$ Gy; bladder $\mathrm{V}_{65}$ $<50 \%$; femur head $\mathrm{V}_{50} \leq 5 \%$ (18).

Preoperative concurrent chemotherapy included capecitabine $\left(825 \mathrm{mg} / \mathrm{m}^{2}\right.$, oral, twice daily) and oxaliplatin $\left(130 \mathrm{mg} / \mathrm{m}^{2}\right.$ on day 1 and day 22$)$ during radiotherapy. Oral capecitabine was given alone to elderly 
patients (age $\geq 65$ ) or to patients who poorly tolerated combination therapy. One cycle CapeOX or two cycles of mFOLFOX6 chemotherapeutic regimens were given after chemoradiotherapy and before radical surgery.

TME surgery was performed 6-8 weeks after neo-CRT and included low anterior resection (LAR), abdominalperineal resection (Miles), and Hartmann's procedure. Adjuvant chemotherapy regimens including CapeOX or mFOLFOX6, and the total perioperative treatment were approximately six months. The specific regimens with dosages were as follows: (I) CapeOX: oxaliplatin $130 \mathrm{mg} / \mathrm{m}^{2}$ $\mathrm{IV}$, day 1 , capecitabine $1,000 \mathrm{mg} / \mathrm{m}^{2}$ twice daily, days $1-14$, every 3 weeks; and (II) mFOLFOX6: oxaliplatin $85 \mathrm{mg} / \mathrm{m}^{2}$ IV day 1 , leucovorin $400 \mathrm{mg} / \mathrm{m}^{2} \mathrm{IV}, 5$-FU $400 \mathrm{mg} / \mathrm{m}^{2} \mathrm{IV}$ bolus on day 1 , then $1,200 \mathrm{mg} / \mathrm{m}^{2} / \mathrm{d} \times 2$ days continuous infusion, every 2 weeks.

\section{Measurement of tumor markers}

Venous blood was collected in the morning before breakfast from all of the subjects. Blood samples were allowed to clot at room temperature for 30 minutes and then centrifuged at $1,000 \mathrm{~g}$ for 20 minutes at $4{ }^{\circ} \mathrm{C}$. The upper phase of the serum was collected, aliquoted, and stored at $-80{ }^{\circ} \mathrm{C}$. Serum CEA and CA19-9 were measured by the electrochemiluminescence immunoassay system Cobas E411 and E601 (Roche, Mannheim, Germany), respectively. The cut-off value is $5 \mathrm{ng} / \mathrm{mL}$ of CEA and $37 \mathrm{U} / \mathrm{mL}$ of CA19-9, as recommended by the manufacturer. Once the tumor markers increased above the upper limit, reexamination would be performed on the same day. The assays were performed blinded to the retrospective study.

\section{Data collection}

A total of 267 eligible patients were retrospectively collected from June 2011 to September 2015. The followup deadline was May 2018, and the median follow-up time was 45.3 months (range, 12.0-86.3 months). Serum levels of CEA and CA19-9 at baseline were detected within one week before neo-CRT. In addition, tumor markers 2-3 weeks after neo-CRT and before radical surgery were also collected for analysis. Sixty-five patients were excluded due to incomplete laboratory results for CEA or CA19-9, and five patients were lost to follow-up, leaving 197 patients available for analysis. All patients were treated with neo-CRT, radical surgery 6-8 weeks later, and adjuvant chemotherapy according to NCCN guidelines. The baseline characteristics of patients are shown in (Table 1), including gender, age, clinical stage, tumor location, baseline values of CEA and CA19-9, CRM (evaluated by MRI), added oxaliplatin or not to concurrent chemoradiotherapy, type of radical surgery and specific downstaging, including $\mathrm{T}$ - and $\mathrm{N}$-downstaging. In addition, clinical staging and corresponding levels of CEA and CA19-9 before and after neo-CRT are shown in (Table S1). Most patients with high levels of CEA or CA19-9 before treatment returned to normal after neo-CRT.

\section{Follow-up}

Patients were followed at 3-month intervals after surgery for two years, at 6-month intervals for the next three years, and once yearly five years later. Evaluations consisted of patient history, physical examination, serum CEA, serum CA19-9, a complete blood count, and blood chemical analysis at every visit. Proctoscopy, abdominal ultrasonography and CT/MRI studies of the abdomen, pelvis, and chest were routinely examined every 6-12 months.

\section{Statistical analysis}

Primary endpoints included OS and disease-free survival (DFS). OS was defined as the time from the beginning of neo-adjuvant chemoradiation to death from any cause. DFS was defined as the time from the start of treatment to the first incidence of either LR or DM. Secondary endpoints included DM and LR. DM was defined as any recurrence outside the pelvic cavity, and LR was defined as any recurrence within the pelvic cavity or perineum.

OS, DFS, DM, and LR rates were estimated utilizing the Kaplan-Meier method and the log-rank test. Multivariate analysis was performed using the Cox proportional hazards regression. Two-sided $\mathrm{P}$ values $<0.05$ were considered statistically significant. All statistical analyses were performed using SPSS software package (version 19.0; IBM, Armonk, NY).

\section{Results}

We first performed survival and recurrence analyses on all patients (OS, DFS, LR, DM), listing all possible variables, including gender, age, tumor location, CRM (evaluated by MRI), CEA, CA19-9, pathologic complete response (pCR), T-downstage, $\mathrm{N}$-downstage, and adding oxaliplatin to the concurrent chemoradiotherapy regimen. 
Table 1 Baseline characteristics of patients

\begin{tabular}{|c|c|c|}
\hline Character & Subtype & $\begin{array}{c}\text { Number of cases } \\
(\%)\end{array}$ \\
\hline \multirow[t]{2}{*}{ Gender } & Male & $133(67.5)$ \\
\hline & Female & $64(32.5)$ \\
\hline \multirow[t]{4}{*}{ Age } & Median [range] & 60 [29-85] \\
\hline & $<50$ & $25(12.7)$ \\
\hline & $50-65$ & $121(61.4)$ \\
\hline & $>65$ & $51(25.9)$ \\
\hline \multirow[t]{5}{*}{ Clinical stage } & II: $\mathrm{cT}_{3-4} \mathrm{~N}_{0} \mathrm{M}_{0}$ & $23(11.7 \%)$ \\
\hline & III A: $\mathrm{cT}_{2} \mathrm{~N}_{1} \mathrm{M}_{0}$ & $12(6.1 \%)$ \\
\hline & III B: $\mathrm{cT}_{3-4} \mathrm{~N}_{1} \mathrm{M}_{0}$ & $84(42.6 \%)$ \\
\hline & III B: $\mathrm{cT}_{2} \mathrm{~N}_{2} \mathrm{M}_{0}$ & $4(2.0 \%)$ \\
\hline & III C: $\mathrm{cT}_{3-4} \mathrm{~N}_{2} \mathrm{M}_{0}$ & 74 (37.6\%) \\
\hline \multirow[t]{3}{*}{ Tumor location } & $<5 \mathrm{~cm}$ & $73(37.1)$ \\
\hline & $5-10 \mathrm{~cm}$ & $110(55.8)$ \\
\hline & $>10 \mathrm{~cm}$ & $14(7.1)$ \\
\hline \multirow[t]{2}{*}{ CEA (ng/mL) } & $\leq 5$ & $113(57.4)$ \\
\hline & $>5$ & $84(42.6)$ \\
\hline \multirow[t]{2}{*}{ CA 19-9 (U/mL) } & $\leq 37$ & $176(89.3)$ \\
\hline & $>37$ & $21(10.7)$ \\
\hline \multirow[t]{2}{*}{ CRM (by MRI) } & Involved & $62(31.5)$ \\
\hline & Clear & $135(68.5)$ \\
\hline \multirow[t]{2}{*}{ CRT with OX } & With & $162(82.2)$ \\
\hline & Without & $35(17.8)$ \\
\hline \multirow[t]{3}{*}{ Type of surgery } & Anterior resection & $123(62.4)$ \\
\hline & Abdominal-perineal resection & $56(28.4)$ \\
\hline & Hartmann & $18(9.1)$ \\
\hline \multirow[t]{2}{*}{ Downstage $^{\S}$} & T-downstage & $108(54.8)$ \\
\hline & N-downstage & 163 (82.7) \\
\hline
\end{tabular}

$\S$, downstage, including T-downstage and $\mathrm{N}$-downstage, means clinical restaging after neo-CRT and before radical surgery compared with clinical staging at initial treatment. CEA, carcinoembryonic antigen; CA19-9, carbohydrate antigen 19-9; CRT, neoadjuvant chemoradiotherapy; OX, oxaliplatin; CRM, circumferential resection margin.

Univariate analysis was performed to identify potential significant variables for different endpoints (OS, DFS, LR, and $\mathrm{DM}$ ) (Table 2), using $\mathrm{P}>0.15$ as the exclusion criterion.
Multivariate analysis was subsequently performed for OS, DFS, LR, and DM (Table 3), which identified independent prognostic risk factors and strongly identified CEA and CA19-9.

\section{Survival and progression analysis}

The median follow-up time was 45.3 months (range, 12.0-86.3 months). A total of 28 patients died, and the 3and 5 -year OS rates were $89.1 \%$ and $82.7 \%$, respectively. Thirteen patients experienced LR (6.6\%), and the 3- and 5 -year LR rates were $5.6 \%$ and $7.2 \%$, respectively. DM occurred in thirty-eight cases $(19.3 \%)$, of which twelve cases (31.6\%) had liver metastases, and 18 (47.4\%) had lung metastases as the first site. The 3- and 5-year DM rates were $18.2 \%$ and $20.3 \%$, respectively. Most local or distant progression occurred within three years, and the 3 - and 5 -year DFS rates were $80.8 \%$ and $78 \%$, respectively.

In univariate analysis, potential predictors for OS included gender, CRM, CEA, CA19-9 and pCR. CRM, CEA, CA19-9, pCR, T-downstage, and N-downstage were the potential prognostic factors for DFS. Potential predictors for LR included tumor location, CA19-9, and pCR; CRM, CEA, CA19-9, pCR, and T-downstage were the potential risk factors for DM, respectively.

By adjusting for all known covariates and using COX regression model analysis, only CRM and CA19-9 were independently predictive of OS. CEA, CA19-9, and pCR were the independent prognostic factors for DFS. CA19-9 and tumor location were predictive of LR; CEA, CA19-9, and $\mathrm{pCR}$ were the independent risk factors for DM. In addition, LARC patients could not benefit from adding oxaliplatin to neo-CRT in the study.

\section{CEA is an independent predictor of DFS and DM}

In the univariate analysis, elevated baseline CEA was associated with reduced OS (5-year, $73.6 \%$ vs. $89.8 \%$, $\mathrm{P}=0.014)$, DFS (5-year, $68.6 \%$ vs. $85.7 \%, \mathrm{P}=0.002$ ), and $\mathrm{DM}(30.2 \%$ vs. $12.1 \%, \mathrm{P}=0.001)$ (Table 2). Kaplan-Meier curves showed a comparison of the OS, DFS, LR, and DM for the elevated and normal CEA levels before treatment in LARC patients with neo-CRT, radical surgery, and adjuvant chemotherapy (Figure 1A,B,C,D). Multivariate analysis suggested patients with elevated CEA were associated with a decreased DFS [hazard ratio (HR): 2.058, 95\% confidence interval (CI): 1.034-4.096, $\mathrm{P}=0.040]$ and a higher risk of DM (HR: 2.144, 95\% CI: 1.058-4.346, $\mathrm{P}=0.034$ ) (Table 3). 
Table 2 Univariate analysis of the effects of different characteristics on 5-year OS, 5-year DFS, DM, and LR

\begin{tabular}{|c|c|c|c|c|c|c|c|c|c|c|}
\hline Characteristic & Subtype & $\mathrm{N}$ & \multicolumn{2}{|c|}{ OS } & \multicolumn{2}{|c|}{ DFS } & \multicolumn{2}{|c|}{ DM } & \multicolumn{2}{|c|}{ LR } \\
\hline \multirow{2}{*}{ Gender } & Male & 133 & 78.3 & \multirow{2}{*}{$0.072^{*}$} & 76.4 & \multirow{2}{*}{0.208} & 22.1 & \multirow{2}{*}{0.197} & 6.8 & \multirow{2}{*}{0.858} \\
\hline & Female & 64 & 91.1 & & 82.1 & & 15.4 & & 7.9 & \\
\hline \multirow{2}{*}{ Age } & $<50$ & 25 & 88 & \multirow{2}{*}{0.854} & 84 & \multirow{2}{*}{0.764} & 16.0 & \multirow{2}{*}{0.847} & 4 & \multirow{2}{*}{0.804} \\
\hline & $>65$ & 51 & 82.3 & & 76.5 & & 21.6 & & 8 & \\
\hline \multirow{2}{*}{ CRM (by MRI) } & + & 62 & 74.5 & \multirow{2}{*}{$0.024^{*}$} & 67.7 & \multirow{2}{*}{$0.012^{\star}$} & 31.1 & \multirow{2}{*}{$0.013^{\star}$} & 11.3 & \multirow{2}{*}{0.174} \\
\hline & - & 135 & 89.5 & & 83.1 & & 15.1 & & 5.6 & \\
\hline Tumor location $(\mathrm{cm})$ & $<5$ & 73 & 79.5 & 0.519 & 73 & 0.401 & 23.8 & 0.549 & 13.6 & $0.041^{*}$ \\
\hline \multirow{2}{*}{ CEA (ng/mL) } & $\leq 5$ & 113 & 89.8 & \multirow{2}{*}{$0.014^{*}$} & 85.7 & \multirow{2}{*}{$0.002^{*}$} & 12.1 & \multirow{2}{*}{$0.001^{\star}$} & 6.2 & \multirow{2}{*}{0.378} \\
\hline & $>5$ & 84 & 73.6 & & 68.6 & & 30.2 & & 8.4 & \\
\hline & $\leq 37$ & 176 & 86.2 & & 82.1 & & 15.9 & & 5.7 & \\
\hline CA19-9 (IU/mL) & $>37$ & 21 & 45.8 & $<0.001^{*}$ & 47.6 & $<0.001^{*}$ & 52.4 & $<0.001^{\star}$ & 19 & $0.006^{\star}$ \\
\hline Nen-CRT with OX & With & 162 & 83.2 & 0.404 & 78.1 & 0923 & 19.8 & 0897 & 6.8 & 0.572 \\
\hline & Without & 35 & 82.8 & & 80 & & 20 & & 8.6 & \\
\hline & Yes & 69 & 90.4 & & 92.8 & & 7.2 & & 2.9 & \\
\hline PCR & No & 128 & 79 & $0.077^{n}$ & 70.9 & $0.001 \times$ & 26.5 & $0.002^{n}$ & 9.4 & $0.144^{\circ}$ \\
\hline
\end{tabular}

*, indicate $\mathrm{P}<0.15 .{ }^{\S}$, downstage, including T-downstage and $\mathrm{N}$-downstage, means clinical restaging after neo-CRT and before radical surgery compared with clinical staging at initial treatment. OS, overall survival; DFS, disease-free survival; LR, local recurrence; DM, distant metastasis; CEA, carcinoembryonic antigen; CA19-9, carbohydrate antigen 19-9; neo-CRT, neoadjuvant chemoradiotherapy. OX, oxaliplatin; CRM, circumferential resection margin; $\mathrm{pCR}$, pathologic complete response; $\mathrm{Cl}$, confidence interval; $\mathrm{T}$, tumor; $\mathrm{N}$, lymph node.

The results indicated that CEA was an independent prognostic risk factor of DFS and DM for LARC patients.

\section{CA19-9 is an independent predictor of OS, DFS, LR, and $D M$}

Compared with normal baseline CA19-9 values, patients with elevated CA19-9 values showed reduced OS (5-year, 45.8\% vs. $86.2 \%, \mathrm{P}<0.001$ ) and DFS ( 5 -year, $47.6 \%$ vs. $82.1 \%$, $\mathrm{P}<0.001)$, along with higher risk of LR $(19 \%$ vs. $5.7 \%$, $\mathrm{P}=0.006)$ and $\mathrm{DM}(52.4 \%$ vs. $15.9 \%, \mathrm{P}<0.001)$ (Table 2$)$.
Kaplan-Meier curves comparing the OS, DFS, LR, and DM of LARC patients with elevated and normal baseline CA19-9 levels after receiving neo-CRT, radical surgery, and adjuvant chemotherapy (Figure $2 A, B, C, D$ ).

The Cox regression model also suggested reduced OS (HR: 2.894, 95\% CI: 1.196-7.006, P=0.018), DFS (HR: 4.533, 95\% CI: 2.067-9.940, $\mathrm{P}<0.001)$, and increased incidence of LR (HR: 6.139, 95\% CI: 1.813-20.783, $\mathrm{P}=0.004)$ and DM (HR: 4.052, 95\% CI: 1.892-8.678, $\mathrm{P}<0.001)$ in LARC patients with elevated baseline CA19-9 levels (Table 3). Therefore, the results above indicated that 
Table 3 Multivariable analysis of different characteristics on 5-year OS, DFS, LR, DM

\begin{tabular}{|c|c|c|c|c|}
\hline Characteristic & Subtype & $\mathrm{P}$ & HR value & $95 \% \mathrm{Cl}$ \\
\hline \multirow{3}{*}{ OS } & CRM (+) & $0.021^{*}$ & 1.659 & $0.763-3.609$ \\
\hline & CEA & 0.298 & 1.561 & $0.675-3.609$ \\
\hline & CA19-9 & $0.018^{*}$ & 2.894 & $1.196-7.006$ \\
\hline \multirow{4}{*}{ DFS } & CRM (+) & 0.278 & 1.444 & $0.743-2.805$ \\
\hline & CEA & $0.040^{*}$ & 2.058 & $1.034-4.096$ \\
\hline & CA19-9 & $<0.001^{*}$ & 4.533 & $2.067-9.940$ \\
\hline & $\mathrm{pCR}$ & $0.007^{*}$ & 4.366 & $1.497-12.737$ \\
\hline \multirow{5}{*}{ DM } & CRM (+) & 0.367 & 1.372 & $0.690-2.725$ \\
\hline & CEA & $0.034^{*}$ & 2.144 & $1.058-4.346$ \\
\hline & CA19-9 & $<0.001^{*}$ & 4.052 & $1.892-8.678$ \\
\hline & $\mathrm{pCR}$ & $0.008^{*}$ & 4.345 & $1.480-12.758$ \\
\hline & T-downstage $e^{\S}$ & 0.407 & 1.385 & $0.641-2.990$ \\
\hline \multirow{3}{*}{ LR } & CA19-9 & $0.004^{*}$ & 6.139 & $1.813-20.783$ \\
\hline & Tumor location & $0.021^{*}$ & 0.280 & $0.095-0.827$ \\
\hline & $\mathrm{pCR}$ & 0.188 & 2.758 & $0.609-12.489$ \\
\hline
\end{tabular}

*, indicate $\mathrm{P}<0.05$, suggesting that these variables are independent risk factors for OS, DFS, DM, and LR. ${ }^{\S}$, downstage, including T-downstage and $\mathrm{N}$-downstage, means clinical restaging after neo-CRT and before radical surgery compared with clinical staging at initial treatment. OS, overall survival; DFS, disease-free survival; LR, local recurrence; DM, distant metastasis; CEA, carcinoembryonic antigen; CA19-9, carbohydrate antigen 19-9; neo-CRT, neoadjuvant chemoradiotherapy; CRM, circumferential resection margin; pCR, pathologic complete response; HR, hazard ratio; T, tumor; $\mathrm{N}$, lymph node.

CA19-9 levels before treatment were a powerful predictor of OS, DFS, LR, and DM in LARC patients after receiving neo-CRT, radical surgery, and adjuvant chemotherapy.

\section{Combined CEA with CA19-9 is a stronger predictor of OS, DFS, LR, and DM}

To investigate whether combining CEA and CA19-9 could strongly predict the prognosis of patients. We divided the patients into four groups: (A) normal levels of CEA and CA19-9 ( $\mathrm{n}=106)$; (B) elevated CEA and normal levels of CA19-9 ( $\mathrm{n}=71)$; (C) normal levels of CEA and elevated levels of CA19-9 ( $n=6)$; (D) high levels of both CEA and CA19-9 ( $\mathrm{n}=14)$.

Kaplan-Meier analysis showed that patients in group
D had significant reduced OS (5-year $44.9 \%$ vs. $91.3 \%)$ and DFS (5-year $35.7 \%$ vs. $86.9 \%$ ) and higher risks of LR $(14.3 \%$ vs. $5.6 \%)$ and $\mathrm{DM}(64.3 \%$ vs. $10.9 \%)$ than those in group A (Figure $3 A, B, C, D$ ). The median OS and the median DFS for group D were 49.3 months (95\% CI: 28.802-69.798), and 16.2 months (95\% CI: 5.750-26.650), respectively. Besides, the Cox regression model also showed that patients in group D had the poorest DFS (HR: 8.157, 95\% CI: 3.232-20.591, $\mathrm{P}<0.001)$ and the highest risk of DM (HR: 8.790, 95\% CI: 3.324-23.248, $\mathrm{P}<0.001$ ).

\section{Discussion}

CEA and CA19-9 are the most commonly used tumor markers in gastrointestinal cancer for diagnosis, treatment 

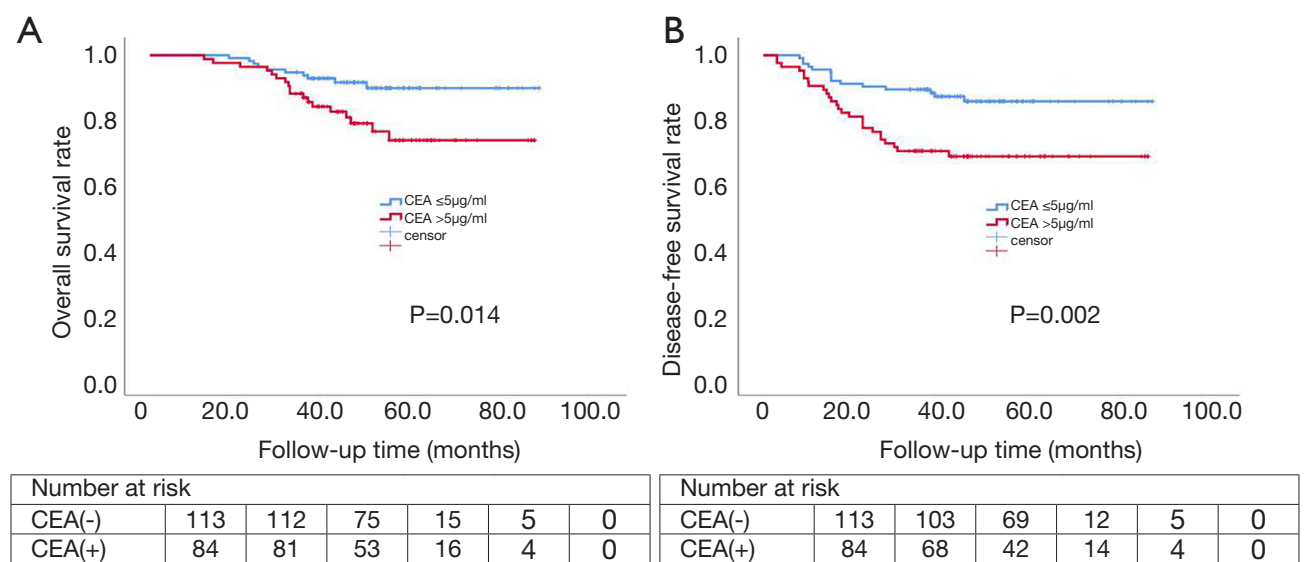

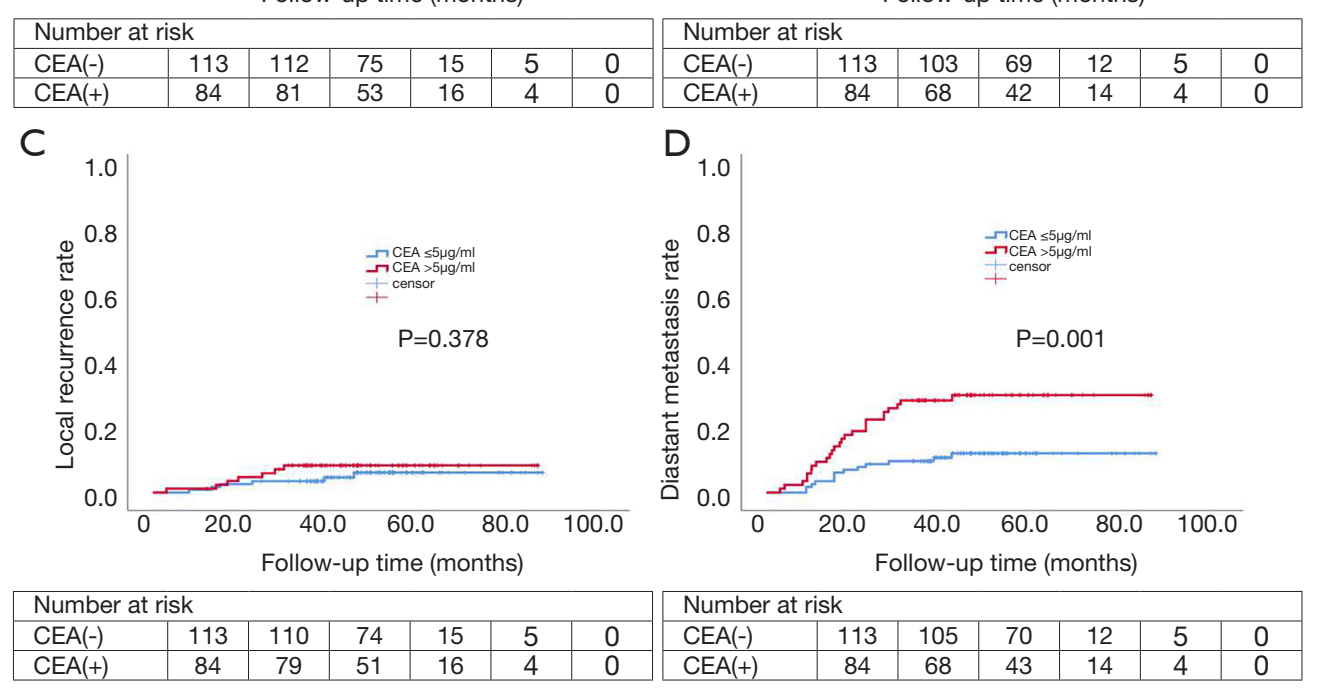

Figure 1 Kaplan-Meier curves comparing the elevated and normal CEA in LARC patients with neo-CRT, radical surgery, and adjuvant chemotherapy. (A) Overall survival; (B) disease-free survival; (C) local recurrence; (D) distant metastasis. LARC, local advanced rectal cancer; CEA, carcinoembryonic antigen. CEA (-): CEA $\leq 5 \mathrm{ng} / \mathrm{mL}$; CEA (+): CEA >5 ng/mL.

assessment, recurrence monitoring, and prognostic evaluation (9-17,19-24). However, in patients with local advanced rectal cancer, CEA and CA19-9 have not been used for clinical decision making. In this study, we found elevated CEA and CA19-9 indicate a poor prognosis, especially for patients with high levels of CA19-9. Although they had received neo-CRT, TME, and postoperative adjuvant chemotherapy, despite most patients with high levels of CEA or CA19-9 at initial treatment returned to normal after neo-CRT. We demonstrated that elevated CEA and CA19-9 are high-risk prognostic factors in LARC patients after the standard first-line therapy.

For LARC patients received neo-CRT, TME, and adjuvant chemotherapy, the available data from our study revealed that the 5 -year OS rate was $82.7 \%$. The survival result was substantially consistent with other randomized trials, which reported rates of approximately $78-81.3 \%$
$(4,25)$. Univariate analysis suggested that elevated CEA was not significantly correlated with LR $(\mathrm{P}=0.378)$, but indicated declined OS, DFS, and DM. Multivariate analysis further showed that elevated CEA is an independent risk factor for DFS and DM. Kim et al. also found CEA was an independent prognostic factor for DFS $(\mathrm{P}=0.004)$ in rectal cancer (26). Restivo et al. demonstrated that CEA was predictive of DM in local advanced rectal cancer (OR 2.107; 95\% CI: 1.313-3.383; $\mathrm{P}=0.002)(27)$.

For patients diagnosed with rectal cancer, CEA was detected as one of the initial evaluation. However, the significance of CA19-9 has not been clear. Zhang et al. reported CA19-9 was the most significant prognostic factor rather than CEA in LARC patients after receiving neoCRT and radical surgery (17). However, $28 \%$ of patients without postoperative chemotherapy in that study. Rectal cancer patients staged as AJCC II/III and all underwent 
A

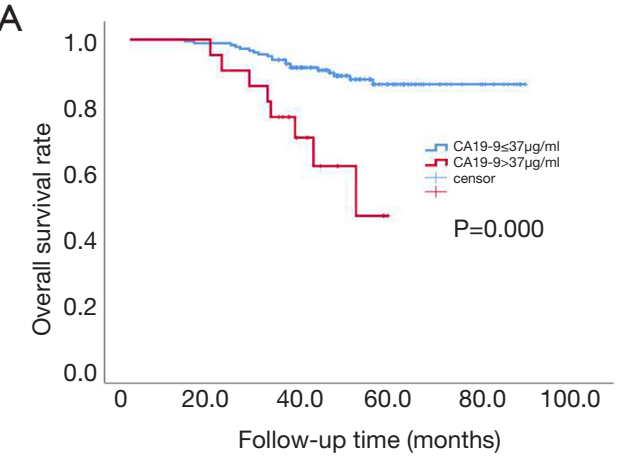

\begin{tabular}{|l|c|c|c|c|c|c|}
\hline Number at risk \\
\hline CA19-9(-) & 176 & 174 & 120 & 31 & 9 & 0 \\
\hline CA19-9(+) & 21 & 19 & 7 & 0 & 0 & 0 \\
\hline
\end{tabular}

C

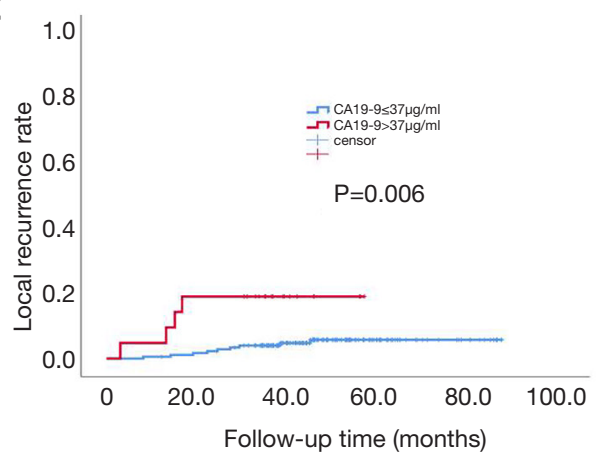

\begin{tabular}{|l|c|c|c|c|c|c|}
\hline \multicolumn{1}{|l|}{ Number at risk } \\
\hline CA19-9(-) & 176 & 172 & 118 & 31 & 9 & 0 \\
\hline CA19-9(+) & 21 & 17 & 6 & 0 & 0 & 0 \\
\hline
\end{tabular}

B

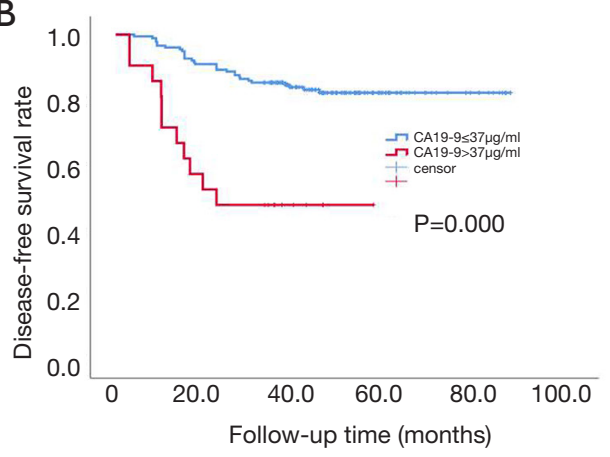

\begin{tabular}{|l|c|c|c|c|c|c|}
\hline \multicolumn{1}{|l|}{ Number at risk } \\
\hline CA19-9(-) & 176 & 160 & 107 & 26 & 9 & 0 \\
\hline CA19-9(+) & 21 & 11 & 4 & 0 & 0 & 0 \\
\hline
\end{tabular}

$\mathrm{D}$

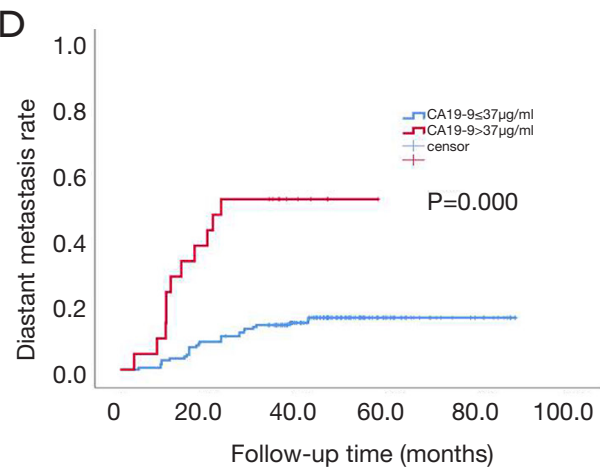

\begin{tabular}{|l|c|c|c|c|c|c|}
\hline \multicolumn{2}{|l|}{ Number at risk } \\
\hline CA19-9(-) & 176 & 161 & 109 & 26 & 9 & 0 \\
\hline CA19-9(+) & 21 & 12 & 4 & 0 & 0 & 0 \\
\hline
\end{tabular}

Figure 2 Kaplan-Meier curves comparing the elevated and normal CA19-9 in LARC patients with neo-CRT, radical surgery, and adjuvant chemotherapy. (A) Overall survival; (B) disease-free survival; (C) local recurrence; (D) distant metastasis. LARC, local advanced rectal cancer; CA19-9, carbohydrate antigen 19-9. CA19-9(-): CA19-9 $\leq 37 \mathrm{U} / \mathrm{mL}$; CA19-9 (+): CA19-9 >37 U/mL.

rigid first-line standard treatment were included for analysis, we found that in addition to the elevated CA19-9, which indicated extremely poor prognosis, the more important thing is that elevated CEA is also a predictor of DFS and DM, and combined CEA with CA19-9 is a better prognostic predictor. Even though these tumor markers decreased significantly after treatment (Table S1), and conventional imaging examinations have ruled out distant metastases before treatment. Herein, we recommend CA19-9 should be routinely included in the initial assessment in addition to CEA for patients diagnosed with local advanced rectal cancer. More intensive treatment or additional treatment strategies may be required, including intensified preoperative chemotherapy, the addition of targeted therapy to preoperative treatment, adjustment of postoperative chemotherapy to preoperative to strengthen systemic control, or genetic testing to select suitable treatment options.

In our retrospective study, MRI-involved CRM was an independent risk factor for OS. It was consistent with the result of the MERCURY trial, where MRI-involved CRM showed a decreased OS in comparison with MRIclear CRM (HR: 1.97, 95\% CI: 1.27-3.04, P<0.01) (28). However, we didn't observe that patients with MRIinvolved CRM had a higher risk of LR. It may because part of patients in the MERCURY trial without neoadjuvant treatment. Besides, we also explored the role of oxaliplatin to neo-CRT. The COX regression model suggested it did not improve local or systemic control significantly. Though, it has been reported in previous well-known phase III randomized clinical trials (RCT) (29-31).

There are some limitations in our study. Although we carried out an explicit enter criterion, a retrospective study with the possibility of involving in confounding 

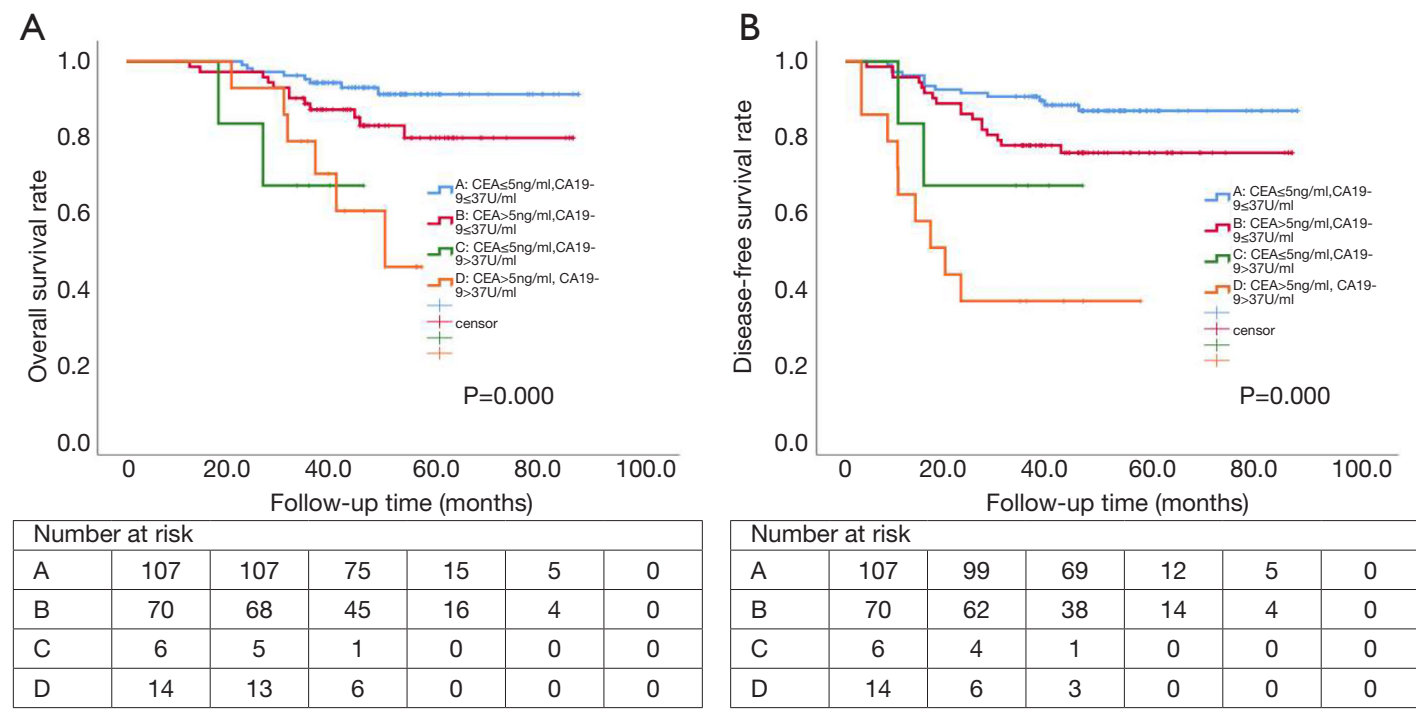

\begin{tabular}{|l|c|c|c|c|c|c|}
\hline \multicolumn{6}{|l|}{ Number at risk } \\
\hline A & 107 & 107 & 75 & 15 & 5 & 0 \\
\hline B & 70 & 68 & 45 & 16 & 4 & 0 \\
\hline C & 6 & 5 & 1 & 0 & 0 & 0 \\
\hline D & 14 & 13 & 6 & 0 & 0 & 0 \\
\hline
\end{tabular}

\begin{tabular}{|l|c|c|c|c|c|c|}
\hline \multicolumn{7}{|l|}{ Number at risk } \\
\hline A & 107 & 99 & 69 & 12 & 5 & 0 \\
\hline B & 70 & 62 & 38 & 14 & 4 & 0 \\
\hline C & 6 & 4 & 1 & 0 & 0 & 0 \\
\hline D & 14 & 6 & 3 & 0 & 0 & 0 \\
\hline
\end{tabular}

C

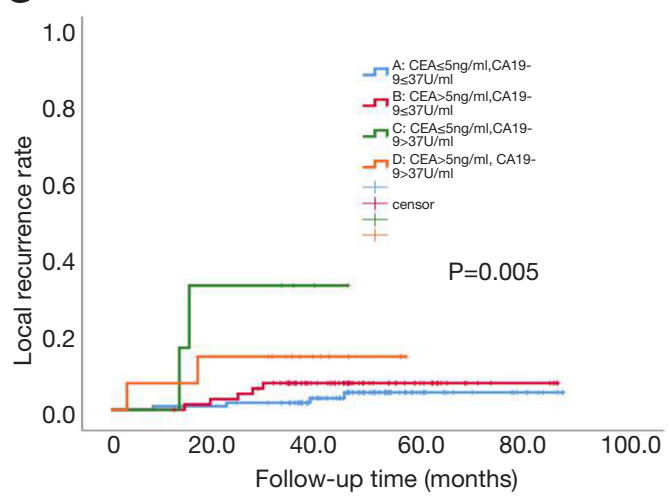

\begin{tabular}{|l|c|c|c|c|c|c|}
\hline \multicolumn{7}{|l|}{ Number at risk } \\
\hline A & 107 & 106 & 74 & 15 & 5 & 0 \\
\hline B & 70 & 67 & 44 & 16 & 4 & 0 \\
\hline C & 6 & 4 & 1 & 0 & 0 & 0 \\
\hline D & 14 & 12 & 6 & 0 & 0 & 0 \\
\hline
\end{tabular}

D

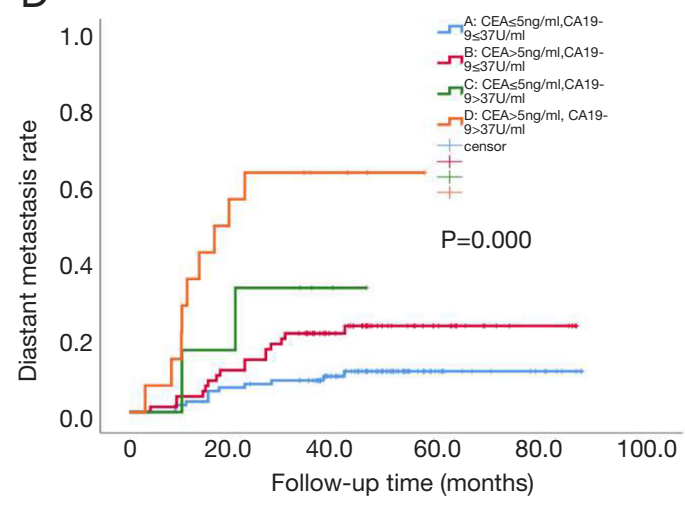

\begin{tabular}{|l|c|c|c|c|c|c|}
\hline \multicolumn{7}{|l|}{ Number at risk } \\
\hline A & 107 & 100 & 70 & 12 & 5 & 0 \\
\hline B & 70 & 62 & 39 & 14 & 4 & 0 \\
\hline C & 6 & 5 & 1 & 0 & 0 & 0 \\
\hline D & 14 & 6 & 3 & 0 & 0 & 0 \\
\hline
\end{tabular}

Figure 3 Kaplan-Meier curves comparing the elevated CEA/CA19-9 in LARC patients with neo-CRT, radical surgery, and adjuvant chemotherapy. (A) Overall survival; (B) disease-free survival; (C) local recurrence; (D) distant metastasis. LARC, local advanced rectal cancer; CEA, carcinoembryonic antigen; CA19-9, carbohydrate antigen 19-9. (A) CEA $\leq 5 \mathrm{ng} / \mathrm{mL}$, CA19-9 $\leq 37 \mathrm{U} / \mathrm{mL}$; (B) CEA >5 ng/mL, CA19-9 $\leq 37 \mathrm{U} / \mathrm{mL}$; (C) CEA $\leq 5 \mathrm{ng} / \mathrm{mL}$, CA19-9 >37 U/mL; (D) CEA >5 ng/mL, CA19-9 >37 U/mL.

factors may result in potential bias. Besides, we formulated strict inclusion in order to reduce interfering factors. Only patients who are accurately staging as AJCC II/III preoperatively and receiving standard neo-CRT combined with TME and postoperative chemotherapy are eligible for further researches, which in turn to limit the sample size, especially for patients with elevated CA19-9. We will expand the sample size for further research. Reanalysis using existing tumor markers or updated data from tumor markers derived from tumor specimens would be of interest in the future.

\section{Conclusions}

To summarize, LARC patients with high levels of CEA or/and CA19-9 before treatment exhibit worse prognosis, despite receiving subsequent radical resection and existing standard adjuvant chemotherapy. Additional studies are 
warranted to further determine the clinical significance of CEA and CA19-9 in rectal cancer. Still, these findings suggest that this patient population needs more intensive treatment or additional treatment strategies.

\section{Acknowledgments}

We thank American Journal Experts for providing professional English language editing.

Funding: This work was supported by grants from the Medical and Health Research Program of Zhejiang Province (No. 2018RC049).

\section{Footnote}

Reporting Checklist: The authors have completed the REMARK reporting checklist. Available at http://dx.doi. org/10.21037/tcr-20-2269

Data Sharing Statement: Available at http://dx.doi. org/10.21037/tcr-20-2269

Peer Review File: Available at http://dx.doi.org/10.21037/tcr20-2269

Conflicts of Interest: All authors have completed the ICMJE uniform disclosure form (available at http://dx.doi. org/10.21037/tcr-20-2269). The authors have no conflicts of interest to declare.

Ethical Statement: The authors are accountable for all aspects of the work in ensuring that questions related to the accuracy or integrity of any part of the work are appropriately investigated and resolved. The study was conducted in accordance with the Declaration of Helsinki (as revised in 2013). The retrospective study was approved by the ethic committee of Sir Run Run Shaw Hospital, School of Medicine, Zhejiang University (Project number 20180927-1). Informed consent was taken from all the patients.

Open Access Statement: This is an Open Access article distributed in accordance with the Creative Commons Attribution-NonCommercial-NoDerivs 4.0 International License (CC BY-NC-ND 4.0), which permits the noncommercial replication and distribution of the article with the strict proviso that no changes or edits are made and the original work is properly cited (including links to both the formal publication through the relevant DOI and the license). See: https://creativecommons.org/licenses/by-nc-nd/4.0/.

\section{References}

1. Glynne-Jones R, Wyrwicz L, Tiret E, et al. Rectal cancer: ESMO Clinical Practice Guidelines for diagnosis, treatment and follow-up. Ann oncol 2018;29:iv263.

2. Khrizman P, Niland JC, ter Veer A, et al. Postoperative adjuvant chemotherapy use in patients with stage II/III rectal cancer treated with neoadjuvant therapy: a national comprehensive cancer network analysis. J Clin Oncol 2013;31:30-8.

3. Benson AB, Venook AP, Al-Hawary MM, et al. Rectal Cancer, Version 2.2018, NCCN Clinical Practice Guidelines in Oncology. J Natl Compr Canc Netw 2018;16:874-901.

4. Fernandez-Martos C, Garcia-Albeniz X, Pericay $\mathrm{C}$, et al. Chemoradiation, surgery and adjuvant chemotherapy versus induction chemotherapy followed by chemoradiation and surgery: long-term results of the Spanish GCR-3 phase II randomized trialdagger. Ann Oncol 2015;26:1722-8.

5. Rodel C, Graeven U, Fietkau R, et al. Oxaliplatin added to fluorouracil-based preoperative chemoradiotherapy and postoperative chemotherapy of locally advanced rectal cancer (the German CAO/ARO/AIO-04 study): final results of the multicentre, open-label, randomised, phase 3 trial. Lancet Oncol 2015;16:979-89.

6. Sauer R, Becker H, Hohenberger W, et al. Preoperative versus postoperative chemoradiotherapy for rectal cancer. N Engl J Med 2004;351:1731-40.

7. Bosset JF, Collette L, Calais G, et al. Chemotherapy with preoperative radiotherapy in rectal cancer. $\mathrm{N}$ Engl J Med 2006;355:1114-23.

8. Sauer R, Liersch T, Merkel S, et al. Preoperative versus postoperative chemoradiotherapy for locally advanced rectal cancer: results of the German CAO/ARO/AIO-94 randomized phase III trial after a median follow-up of 11 years. J Clin Oncol 2012;30:1926-33.

9. Nakayama T, Watanabe M, Teramoto T, et al. CA19-9 as a predictor of recurrence in patients with colorectal cancer. J Surg Oncol 1997;66:238-43.

10. Shibutani M, Maeda K, Nagahara H, et al. Significance of CEA and CA19-9 combination as a prognostic indicator and for recurrence monitoring in patients with stage II colorectal cancer. Anticancer Res 2014;34:3753-8.

11. Tokunaga R, Sakamoto Y, Nakagawa S, et al. The utility of 
tumor marker combination, including serum P53 antibody, in colorectal cancer treatment. Surg Today 2017;47:636-42.

12. Verazin G, Riley WM, Gregory J, et al. Serum sialic acid and carcinoembryonic levels in the detection and monitoring of colorectal cancer. Dis Colon Rectum 1990;33:139-42.

13. Yang W, Luo Y, Hu S, et al. Value of combined detection of serum carcino-embryonic antigen, carbohydrate antigen 19-9 and cyclooxygenase-2 in the diagnosis of colorectal cancer. Oncol Lett 2018;16:1551-6.

14. Cai D, Huang ZH, Yu HC, et al. Prognostic value of preoperative carcinoembryonic antigen/tumor size in rectal cancer. World J Gastroenterol 2019;25:4945-58.

15. Weissenberger C, Von Plehn G, Otto F, et al. Adjuvant radiochemotherapy of stage II and III rectal adenocarcinoma: role of CEA and CA 19-9. Anticancer Res 2005;25:1787-93.

16. Yang KL, Yang SH, Liang WY, et al. Carcinoembryonic antigen (CEA) level, CEA ratio, and treatment outcome of rectal cancer patients receiving pre-operative chemoradiation and surgery. Radiat Oncol 2013;8:43.

17. Zhang LN, OuYang PY, Xiao WW, et al. Elevated CA19-9 as the Most Significant Prognostic Factor in Locally Advanced Rectal Cancer Following Neoadjuvant Chemoradiotherapy. Medicine (Baltimore) 2015;94:e1793.

18. Emami B, Lyman J, Brown A, et al. Tolerance of normal tissue to therapeutic irradiation. Int J Radiat Oncol Biol Phys 1991;21:109-22.

19. Basbug M, Arikanoglu Z, Bulbuller N, et al. Prognostic value of preoperative CEA and CA 19-9 levels in patients with colorectal cancer. Hepatogastroenterology 2011;58:400-5.

20. Duffy MJ. Role of tumor markers in patients with solid cancers: A critical review. Eur J Intern Med 2007;18:175-84.

21. Kochi M, Fujii M, Kanamori N, et al. Evaluation of serum CEA and CA19-9 levels as prognostic factors in patients with gastric cancer. Gastric Cancer 2000;3:177-86.

22. Sisik A, Kaya M, Bas G, et al. CEA and CA 19-9 are still valuable markers for the prognosis of colorectal and gastric cancer patients. Asian Pac J Cancer Prev 2013;14:4289-94.
23. Wang J, Wang X, Yu F, et al. Combined detection of preoperative serum CEA, CA19-9 and CA242 improve prognostic prediction of surgically treated colorectal cancer patients. Int J Clin Exp Pathol 2015;8:14853-63.

24. Zheng CX, Zhan WH, Zhao JZ, et al. The prognostic value of preoperative serum levels of CEA, CA19-9 and CA72-4 in patients with colorectal cancer. World J Gastroenterol 2001;7:431-4.

25. Allegra CJ, Yothers G, O'Connell MJ, et al. Neoadjuvant 5-FU or Capecitabine Plus Radiation With or Without Oxaliplatin in Rectal Cancer Patients: A Phase III Randomized Clinical Trial. J Natl Cancer Inst 2015;107:djv248.

26. Kim CH, Huh JW, Yeom SS, et al. Predictive value of serum and tissue carcinoembryonic antigens for radiologic response and oncologic outcome of rectal cancer. Pathol Res Pract 2020;216:152834.

27. Restivo A, Delrio P, Deidda S, et al. Predictors of Early Distant Relapse in Rectal Cancer Patients Submitted to Preoperative Chemoradiotherapy. Oncol Res Treat 2020;43:146-52.

28. Faletti R, Gatti M, Arezzo A, et al. Preoperative staging of rectal cancer using magnetic resonance imaging: comparison with pathological staging. Minerva Chir 2018;73:13-9.

29. Aschele C, Cionini L, Lonardi S, et al. Primary tumor response to preoperative chemoradiation with or without oxaliplatin in locally advanced rectal cancer: pathologic results of the STAR-01 randomized phase III trial. J Clin Oncol 2011;29:2773-80.

30. Gerard JP, Azria D, Gourgou-Bourgade S, et al. Comparison of two neoadjuvant chemoradiotherapy regimens for locally advanced rectal cancer: results of the phase III trial ACCORD 12/0405-Prodige 2. J Clin Oncol 2010;28:1638-44.

31. O'Connell MJ, Colangelo LH, Beart RW, et al. Capecitabine and oxaliplatin in the preoperative multimodality treatment of rectal cancer: surgical end points from National Surgical Adjuvant Breast and Bowel Project trial R-04. J Clin Oncol 2014;32:1927-34.
Cite this article as: Shan J, Gu B, Shi L, Wang X, Ye W, Zhou W, Sun X. Prognostic value of CEA and CA19-9 in patients with local advanced rectal cancer receiving neoadjuvant chemoradiotherapy, radical surgery and postoperative chemotherapy. Transl Cancer Res 2021;10(1):88-98. doi: $10.21037 /$ tcr-20-2269 
Supplementary

Table S1 Clinical staging and corresponding levels of CEA and CA19-9 before and after neo-CRT

\begin{tabular}{|c|c|c|c|c|c|}
\hline $\begin{array}{l}\text { Clinical } \\
\text { staging }^{\S}\end{array}$ & $\begin{array}{l}\text { Number of cases } \\
(n=197)\end{array}$ & \multicolumn{2}{|c|}{ CEA (+) } & \multicolumn{2}{|c|}{ CA19-9 (+) } \\
\hline cT2 & $16(8.1 \%)$ & $2(2.4 \%)$ & 3 (15.8\%) & $2(9.5 \%)$ & $1(20 \%)$ \\
\hline cT3 & 160 (81.2\%) & 71 (84.5\%) & 15 (78.9\%) & 17 (81.0\%) & $3(60 \%)$ \\
\hline $\mathrm{cN} 1$ & 96 (48.7\%) & 31 (36.9\%) & 6 (31.6\%) & $10(47.6 \%)$ & $3(60 \%)$ \\
\hline $\mathrm{cN}_{2}$ & 78 (39.6\%) & 45 (53.6\%) & $10(52.6 \%)$ & $8(38.1 \%)$ & 2 (40\%) \\
\hline
\end{tabular}

$\S$, clinical restaging after neo-CRT was evaluated by enhanced pelvic MRI. CEA, carcinoembryonic antigen; CA19-9, carbohydrate antigen 19-9; neo-CRT, neoadjuvant chemoradiotherapy. CEA (+): CEA >5 ng/mL, CA19-9 (+): CA19-9 >37 IU/mL. 